\title{
The Effect of AMC for Each Medium on QoE of Video and Audio Transmission over Wireless LANs
}

\author{
Toshiro Nunome ${ }^{1+}$ and Naruhiro Murase $^{2}$ \\ ${ }^{1}$ Department of Computer Science, Graduate School of Engineering \\ ${ }^{2}$ Department of Computer Science, Faculty of Engineering, \\ Nagoya Institute of Technology, Nagoya 466-8555, Japan
}

\begin{abstract}
In this paper, we assess QoE of video and audio transmission over wireless LANs. This study tries to employ different AMC mechanis m for each med ium. We consider four AMC methods: ARF, AARF, AARF-CD, and CARA. Through computer simulation and subjective experiment, we notice that the appropriate $\mathrm{AMC}$ for the required quality can enhance QoE.
\end{abstract}

Keywords: wireless LAN, streaming, QoE, AMC

\section{Introduction}

Wireless LANs (Local Area Networks) are indispensable for our daily lives today. Multimedia communications with video and audio transmission have usually been performed over the wireless LANs. Packet loss and delay jitter degrade QoS (Quality of Service) and QoE (Quality of Experience) [1] because the wireless channel is frequently unstable.

AMC (Adaptive Modulation and Coding) is employed for efficient transmission over wireless channels. It adopts the coding rate to the wireless channel conditions. Many studies have been done on AMC [2]-[5]. There are various rate adaptation algorithms. In general, AMC controls transmission rate for device-todevice communications; all the streams are transmitted with the same AMC strategy.

The main characteristic of multimedia communications is the simultaneous transmission of plural media streams with different features. The feature has various requirements for communications, e.g., delay, delay jitter, and reliability. For example, in [6], we investigate an audiovisual spatiotemporal quality tradeoff by means of output schemes of each medium. One is concealment and output of lost portions, and the other is just skipping the portions. The study is performed on an experimental wired network.

To exploit the characteristic of wire less networks and cross-modal effect of multimedia communications, we can consider employing AMC for each medium. Reference [7] considers SVC (Scalable Video Coding) in H.264/AVC video. In SVC, a video stream is encoded into a BL (Base Layer) and multiple ELs (Enhancement Layers). The paper employs different modulation and coding schemes for the layers. But, the paper only considers video streams and then does not exploit cross-modal effects of different media streams.

$\mathrm{QoE}$ is an ultimate performance measure for the users. The evaluation of the network services has to be done at the QoE-level.

In this study, we assess QoE of video and audio transmission over wireless LANs with different AMC mechanism for each medium.

\footnotetext{
${ }^{+}$Corresponding author. Tel.: +81-52-735-7785; fax: +81-52-735-5442.
}

E-mail address:nunome@nitech.ac.jp. 
The remainder of this paper is organized as follows. Section 2 introduces the AMC mechanisms employed in this paper. Section 3 describes the simulation and the QoE assessment method. Section 4 presents experimental results. Section 5 concludes this paper.

\section{AMC Mechanisms}

In this study, we employ four AMC methods: ARF (Auto Rate Fallback) [2], AARF (Adaptive ARF) [3], AARF-CD (AARF with Collision Detection) [4], and CARA (Collision Aware Rate Adaptation) [5]. The summaries of the methods are as follows.

ARF increases the transmission rate according to the number of successive successful transmissions. When it successes ten successive transmissions, it increases the transmission rate. On the other hand, the transmission rate decreases when the transmission is failed successively twice.

AARF modifies the threshold for the number of successive successful transmissions in ARF. AARF increases the threshold twice when the transmission just after the rate increase is failed.

AARF-CD introduces RTS/CTS (Request to Send/Clear to Send) into AARF.

CARA employs RTS/CTS in addition to original ARF.

\section{Methodology of Performance Evaluation}

\subsection{Simulation}

The network topology is shown in Fig. 1. AP and router (R) are connected through a P2P link. Five wired nodes (WN) are connected to R with CSMA links; we employ the link to assume Ethernet links. The bitrate of both P2P link and CSMA links is 100 Mbps. The propagation de lay is $1 \mathrm{~ms}$. Six mobile nodes (MN) are connected to AP via an IEEE 802.11g wireless LAN. The distance between AP and each MN is set to $r$ m. As the distance $r$ increases, the channel condition becomes worse.

$\mathrm{WN}_{6}$ transmits audio and video streams to $\mathrm{MN}_{6}$ using RTP/UDP/IP. We refer to a unit for media synchronization control as a Media Unit (MU); a video frame is defined as a video MU, and a constant number of audio samples is called as an audio MU. A video frame consists of 5 video slices, and each slice is transmitted by an RTP packet. An audio MU is transmitted by an RTP packet.

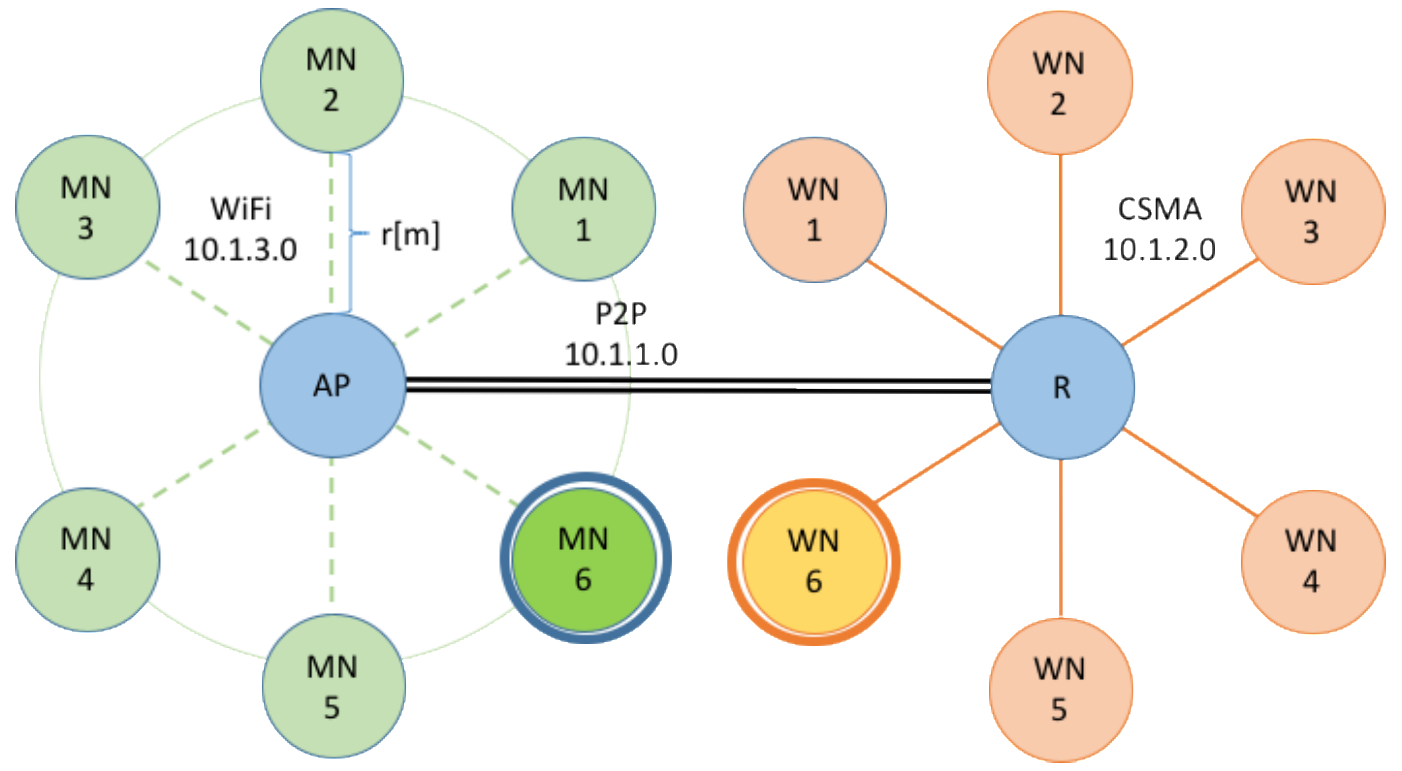

Fig. 1: Network topology

The specifications of video and audio are as follows. The contents are "sport" and "music". "sport" is a video dominant content, while "music" is an audio dominant content. The video is encoded to H.264/AVC by JM 16.2. The audio is linear PCM (monaural, $12 \mathrm{kHz}, 16 \mathrm{bit}$ ). The image size is $320 \times 240$ pixels. The average bitrate of video is $775.7 \mathrm{kbps}$ in "sport" and $803.2 \mathrm{kbps}$ in "music". The average bitrate of audio is 192 kbps. 
As a media synchronization control, we employ a simple playout buffering control for each media. The receiver outputs audio and video after the playout buffering control for absorbing network delay jitter. The playout buffering time is set to $2000 \mathrm{~ms}$ in this paper.

Wired nodes $\mathrm{WN}_{k}(\mathrm{k}=1,2,3,4,5)$ and mobile nodes $\mathrm{MN}_{k}$ are used to handle background traffic flows for the audio and video streams. A pair of $\mathrm{WN}_{k}$ and $\mathrm{MN}_{k}$ is referred to as a load terminal pair. The nodes generate fixed-size IP datagrams of 1500 bytes each at exponentially distributed intervals. The amount of the traffic is adjusted by changing the average of the interval. We refer to the average amount of the traffic for each load terminal as the average load. In this study, we set that the average load for each downlink load terminal $\mathrm{WN}_{k}$ is $800 \mathrm{kbps}$, and that for each uplink load terminal $\mathrm{MN}_{k}$ is $400 \mathrm{kbps}$.

For each combination of the following parameters, we perform 15 simulation runs.

Contents (two types): sport, music

AMC for audio (four types): ARF, AARF, AARF-CD, CARA

AMC for video (four types): ARF, AARF, AARF-CD, CARA

Distance between AP and each terminal (ten types):

45 m, 55 m, 65 m, 75 m, 85 m, 90 m, 92 m, 94 m, 95 m, 96 m

\subsection{QoE assessment}

In this paper, we assess QoE of the audio-video stream transferred with the simulation parameters described in the previous section by a subjective experiment. It was conducted as follows.

From the simulation results, we notice that the application-level QoS, which is closely related to QoE, for the distance $45 \mathrm{~m}$ through $75 \mathrm{~m}$ has minor differences. Thus, we employ the distance between $85 \mathrm{~m}$ though $96 \mathrm{~m}$ for QoE assessment.

A subjective score is measured by the rating-scale method, in which assessors classify each stimulus into one of a certain number of categories. We adopted the five categories of impairment as shown in Table 1 . The integer value is regarded as a subjective score. We then calculate mean opinion score (MOS) as the quantitative measure of perceptual quality.

The assessors are 15 male students in their twenties. Each stimulus lasted 10 seconds and was obtained by outputting the audio-video stream from time 40 to 50 after starting the transmission in the simulation.

Table 1: Five categories of impairment

\begin{tabular}{|l|l|}
\hline \multicolumn{1}{|c|}{ score } & \multicolumn{1}{c|}{ category } \\
\hline 5 & imperceptible \\
\hline 4 & perceptible but not annoying \\
\hline 3 & slightly annoying \\
\hline 2 & annoying \\
\hline 1 & very annoying \\
\hline
\end{tabular}

\section{Results}

Figure 2 depicts the MOS values for "sport". The abscissa means the combination of the distance and the AMC method for audio. The legend represents the AMC method for video.

When we use ARF for audio, we see in Fig. 2 that CARA for video has the larger MOS value than ARF for video in "sport" except for the distance $94 \mathrm{~m}$. CARA can restrict unnecessary rate reduction owing to the collision avoidance mechanism with RTS/CTS, and then CARA has the higher performance in the video dominant content.

As for AARF for audio, we notice in Fig. 2 that the MOS values decrease for the distance larger than 94 $\mathrm{m}$. In the large distances, the audio MU loss is dominant for the MOS values; it is a characteristic of crossmodality of audiovisual transmission. 
We notice in Fig. 2 that for the distance $94 \mathrm{~m}$ and $95 \mathrm{~m}$, AARF-CD for video has the relatively higher MOS values than the other methods when we employ AARF-CD for audio.

When we apply CARA for audio, we find in Fig. 2 that CARA for video has the better MOS values than the other methods except for the distance $96 \mathrm{~m}$. In CARA, using the same AMC method for both media is the best choice.

Figure 3 displays the MOS values for "music". The MOS values for "music" tend to be higher than those for "sport". The results for "music" have basically the same tendency as those for "sport".

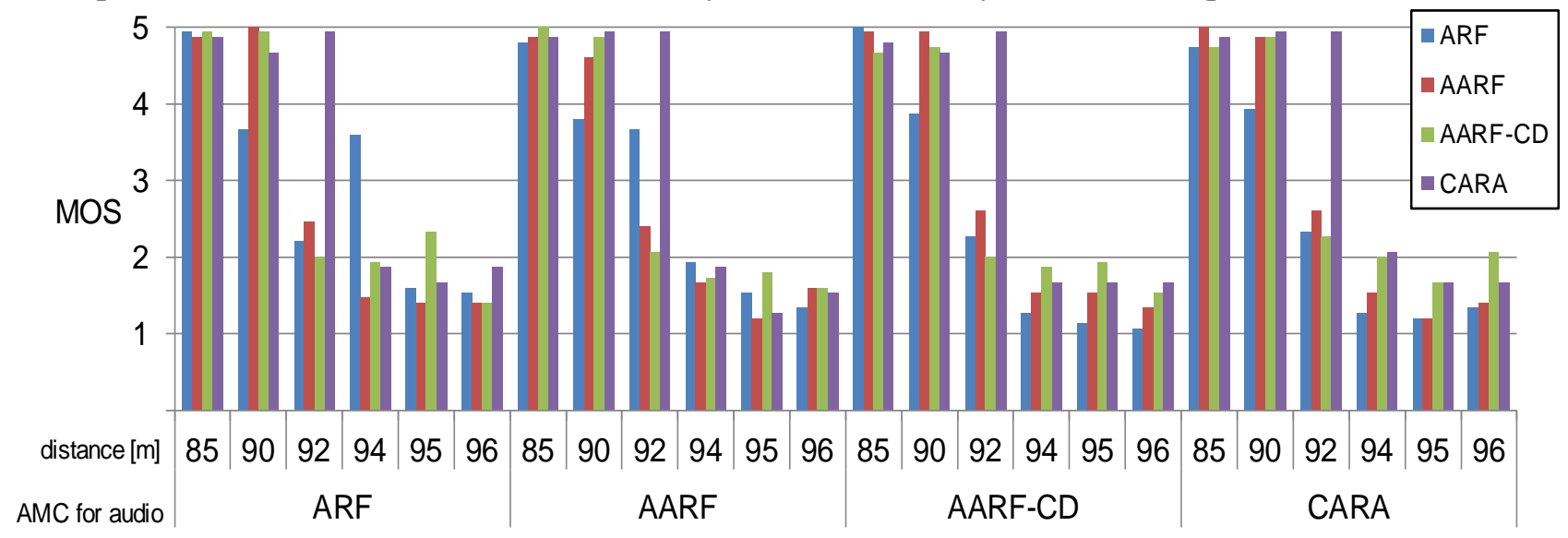

Fig. 2: Mean opinion score for "sport"

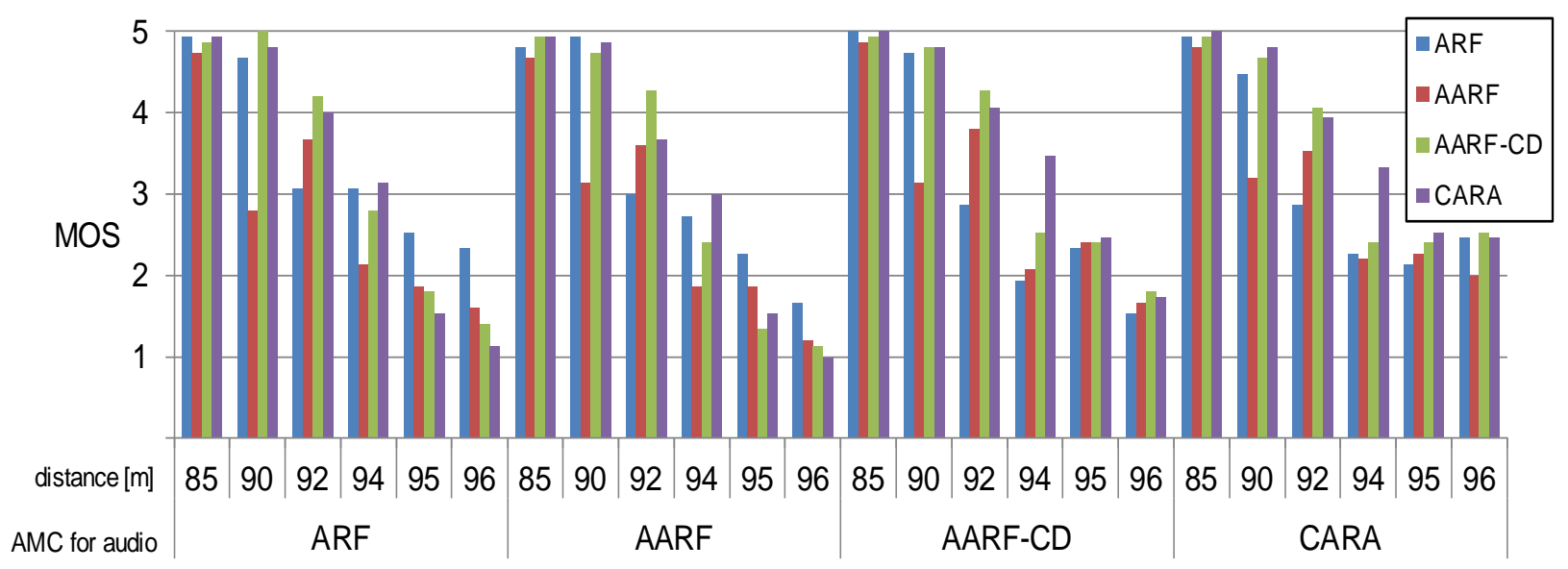

Fig. 3: Mean opinion score for "music"

\section{Conclusions}

In this study, we evaluated QoE of video and audio transmission over wireless LANs with different AMC mechanism for each medium. As a result, we found that the appropriate AMC for the required quality can enhance QoE. For audio, the users require smoothness output, and then CARA is a good choice. On the other hand, for video, the high throughput is required, and then AARF-CD and CARA can achieve higher QoE.

In the future study, we need to assess the effect of other AMC methods. In addition, we also consider other physical layer standards.

\section{References}

[1] ITU-T Rec. P.10/G.100, "Vocabulary for performance, quality of service and quality of experience," Nov. 2017.

[2] A. Kamerman and L. Monteban, "WaveLAN-II: A high-performance wireless LAN for the unlicensed band," Bell Labs Technical Journal, vol. 2, no. 3, pp.118-133, Aug. 1997.

[3] M. Lacage, M. H. Manshaei and T. Turletti, "IEEE 802.11 rate adaptation: A practical approach," Proc. MSWiM '04, pp.126-134, Oct 2004. 
[4] F. Maguolo, "Efficient collision detection for auto rate fallback algorithm," Proc. IEEE ISCC 2008 , July 2008.

[5] J. Kim, S. Kim and S. Choi, "CARA: Collision-aware rate adaptation for IEEE 802.11 WLANs," Proc. IEEE INFOCOM 2006, April 2006.

[6] T. Nunome and H. Ono, "The cross-modal effect of audiovisual interpolation on multimedia QoE over IP network," Far East Journal of Electronics and Communications, vol. 14, no. 1, pp. 15-34, Mar. 2015.

[7] W. -H. Kuo, R. Kaliski, and H. Y. Wei, “A QoE-based link adaptation scheme for H.264/SVC video multicast over IEEE 802.11," IEEE Trans. on Circuits and Syst. for Video Tech., vol. 25, issue 5, pp. 812-826, May 2015. 